\title{
Current and Future Treatment Options in Osteoporosis.
}

\author{
L Brewer, D Williams, A Moore
}

\section{Introduction}

Osteoporosis is a metabolic bone disease that results in bone fragility and increased susceptibility to fracture. As populations age globally, the incidence of osteoporosis-related fractures will rise substantially over the coming decades. Even if age-adjusted incidence rates remain stable, the estimated number of hip fractures worldwide will rise from 1.7 million in 1990 to 6.3 million in $2050 .{ }^{[1]}$ Approximately 1 in 2 women and 1 in 4 men aged over 50 years will have an osteoporosis-related fracture in their lifetime. The increase in medical visits, hospitalisations, need for community supports and nursing home admissions will pose a major economic burden on healthcare systems. Currently the combined annual cost of all osteoporotic fractures has been estimated at $\$ 20$ billion for the US and $\$ 30$ billion for the EU. ${ }^{[2]}$

In order to offer patients greater protection against osteoporosis-related fractures an increasing number of newer therapies have been developed. Knowledge of underlying mechanisms of osteoporosis has advanced in recent years allowing novel agents to be introduced (Figure 1). Bone is continually remodelled to replace microdamage and to adapt bone density to patterns of usage. Agerelated bone loss results in $0.5 \%$ decline in bone mass per year. This occurs because of an imbalance between the activity of osteoclasts (bone-resorbing cells) and osteoblasts (bone-forming cells) and multiple factors are now known to regulate this balance. In this article we briefly summarize the evidence of established treatments before outlining a number of newer agents and prescribing considerations for this common condition.

\section{Determining Fracture Risk}

Prevention of osteoporosis is preferable to treatment as microarchitectural changes associated with bone loss are largely irreversible. Although BMD is the best available non-invasive assessment of bone strength, other factors contribute to bone quality including the mineralisation and rate of bone turnover. ${ }^{[3]}$ Markers of bone formation, turnover and resorption have been used in some trials to assess drug efficacy. Studies also show their highly significant correlation with subsequent rate of 
bone loss and future fracture risk ${ }^{[4]}$ and subgroups with high turnover at baseline have been shown in some studies to gain most from antiresorptive therapy. ${ }^{[5-7]}$

Pharmacological treatments may stabilise or increase BMD but are unlikely to fully restore bone quality and strength. Dual energy x-ray absorptiometry (DXA) is the most widely used method for measuring BMD as it gives very precise measurements at clinically relevant sites. The greatest relative risk of fracture is in individuals with lower BMD but the absolute number of fractures in those with BMD T-scores in the osteopenic range is greater than those with T-scores in the osteoporotic range. Therefore assessment of risk factors that are independent of BMD is important for fracture prediction. ${ }^{[8]}$ Advanced age and previous fracture are the most significant of these validated risk factors (Table II). In 2008 the World Health Organisation (WHO) introduced FRAX ${ }^{\circledR}$, the Fracture Risk Assessment Tool, which estimates the 10 year probability of hip fracture or major osteoporotic fractures combined. ${ }^{[9]}$ It has been validated in 11 independent cohorts and allows estimation of 10 year fracture probability from an individual's set of risk factors alone or in combination with BMD. Patients can be stratified according to fracture risk instead of using BMD alone and intervention can therefore be effectively targeted to those at highest risk of fracture (Figure 2). The FRAX ${ }^{\circledR}$ tool enables the estimation of risk based on a wider range of risk factors, and treatment efficacy is dependent on the level of risk in the analyzed population. Furthermore thresholds for cost-effective pharmacological intervention have been calculated using fracture and economic data that are country-specific. ${ }^{[10]}$ FRAX ${ }^{\circledR}$ can also be used to assess the cost-effectiveness of individual drugs to treat osteoporosis. $[11,12]$

Since the introduction of $F R A X^{\circledR}$, two new fracture clinical risk scores (QFractureScores) have been developed and validated in the UK. ${ }^{[13]}$ In addition to traditional variables already included in the FRAX ${ }^{\circledR}$ algorithm, further fracture risk factors are included such as history of falls, type 2 diabetes, cardiovascular disease, asthma, use of hormone replacement therapy (HRT) and use of tricyclic antidepressants. No BMD score is used in the calculation and patients as young as 30 can be assessed. It can be used to estimate fracture risk at one, two, five, and 10 years. Validation statistics, especially for the hip fracture algorithm, suggest that the QFractureScores are likely to be effective at identifying patients at high risk of fracture and suggested favourable performance compared with FRAX $^{\circledR}$. 
Application of this approach of fracture risk assessment to individual patients allows clinicians to make more informed decisions when considering whether or not to commence pharmacological intervention.

\section{Anti-resorptive agents}

\section{Calcium \& Vitamin D}

Calcium and vitamin $\mathrm{D}$ are necessary for normal skeletal homeostasis and are considered the first step in osteoporosis treatment. Inadequate intake of calcium and vitamin D leads to reduced calcium absorption, a compensatory rise in parathyroid hormone, higher bone remodelling rates and increased bone loss. Vitamin $\mathrm{D}$ deficiency is also linked to reduced muscle function and increased risk of falling and its replacement has been shown to reduce the rate of falling amongst older individuals. ${ }^{[14]}$ The positive association between calcium and vitamin $\mathrm{D}$ levels and bone mineral density (BMD) has been well documented. ${ }^{[15-17]}$ However their replacement, either alone or in combination, has been shown to lower fracture rates in some trials but not in others. Calcium supplementation alone has been shown to reduce the incidence of osteoporotic fracture compared with placebo (HR 0.66) only in patients who are over $80 \%$ compliant. $^{[18]}$

The relationship between vitamin $D$ replacement and fracture prevention is unclear when first examined and many studies using various doses of $25 \mathrm{OHD}$ and differently aged study populations have attempted to clarify this relationship. Despite some inconsistent trial results it appears that vitamin $D$ supplementation studies that had positive results achieved higher vitamin $D$ levels. From available evidence, it seems that individuals will benefit by having their $25 \mathrm{OHD}$ levels no lower than 70 nmol/l. Vitamin D protects against fracture by decreasing PTH and increasing bone mass and also by lowering risk of falling. ${ }^{[19]}$ A higher level may provide additional benefit, but this has not been established. Supplemental calcium, used in many of the trials, both positive and negative, may be essential for benefit but this remains uncertain. The recommended dose of calcium, usually in the form of calcium carbonate, is $1 \mathrm{~g}$ daily and common side effects include dyspepsia and constipation. However an increased risk of myocardial infarction ( $\mathrm{HR} 1.31,95 \% \mathrm{Cl} 1.02$ to 1.67 ) in patients on calcium supplements, without co-prescribed vitamin D, was recently reported in a meta-analysis by Bolland and colleagues ${ }^{[20]}$ which raises concerns about its widespread use. Due to limited available 
evidence of it's benefit when used alone, it is advised that calcium should not be prescribed to patients with osteoporosis unless they are also receiving vitamin $D$ and/or an effective treatment for osteoporosis.

\section{Bisphosphonates}

Bisphosphonates are now widely used in the treatment of osteoporosis as they inhibit bone resorption with relatively few side effects. There are two classes of bisphosphonate: the nitrogen-containing and non nitrogen-containing bisphosphonates all of which have a structure similar to pyrophosphate and thereby inhibit the activation of enzymes that utilise pyrophosphate. ${ }^{[21]}$ Alendronate, risedronate, ibandronate and zoledronic acid are all nitrogen-containing bisphosphontes and have demonstrated efficacy in fracture risk reduction. For these bisphosphonates, the direct intracellular target is the enzyme farnesyl diphosphate synthase in the HMG-CoA reductase biosynthetic pathway. Its inhibition suppresses a process, protein prenylation, resulting in disruption to vital signalling pathways necessary for osteoclastic bone resorption. Bisphophonate molecules preferentially bind to calcium and therefore accumulate to a high concentration in bones where they are ingested by osteoclasts.

Alendronate and risdeonate are most commonly given in weekly oral formulations and zoledronic acid (ZA) is administered annually as an intravenous infusion. Ibandronate comes in monthly oral or 3 monthly intravenous formulations. Alendronate has been studied in postmenopausal women with low $\mathrm{BMD},{ }^{[22,23]}$ with or without prevalent vertebral fractures and subgroup analysis of those women with documented osteoporosis reported a reduction in hip fractures of over $50 \%$. Treatment also demonstrated a reduction of $44-50 \%$ in vertebral fractures. Risedronate also has documented efficacy in improving BMD and fracture risk in postmenopausal women with osteoporosis and vertebral fractures. A reduction in the risk of vertebral and non vertebral fractures of $41 \%$ and $49 \%$ respectively has been reported ${ }^{[24]}$ and in older women (70-79 years) with osteoporosis treatment reduced the rate of hip fracture by $30 \% .{ }^{[25]}$ Positive data on risk reduction of hip and vertebral fracture is also available for Ibandronate, showing similar results to other bisphosphonates. ${ }^{[26,27]}$ Annual ZA reduces vertebral and hip fracture by $70 \%$ and $41 \%$ respectively compared with placebo ${ }^{[28]}$ and offers $35 \%$ relative reduction in recurrent fracture and all-cause mortality (HR 0.72) when given after 2 weeks post hip fracture. ${ }^{[29,30]}$ 
Although oral bisphosphonates are well tolerated if swallowed as indicated (Table 1), caution is advised with a background of gastrointestinal complaints. Recent reports have proposed a link between bisphosphonate-related oesophagitis and oesophageal cancer ${ }^{[31]}$ and this association has been investigated using data from the UK General Practice Research database and reported in two separate studies. At a follow-up period of four and a half years, Cardwell et al reported no increase in the risk of oesophageal or gastric cancer with bisphosphonate use. ${ }^{[32]}$ In contrast the case-control analysis by Green et al. ${ }^{[33]}$ found an increase in the risk of oesophageal cancer with 10 or more prescriptions for oral bisphosphonates and with prescriptions over a five year period (an increase in incidence of 1 per 1000 to 2 per 1000 population aged 60-79 years with five years' use of oral bisphosphonates). This study had a longer follow-up period of 7.7 years. However, neither study validated diagnoses by medical records nor provided information on whether drugs were taken according to directions. Even if oral bisphosphonates do increase the risk of oesophageal cancer, the incidence in the population would be expected to remain relatively low. As evidence to date is inconclusive, cautious prescribing of bisphosphonates, patient education and active follow-up are advised.

Adverse skeletal events in the form of atypical fragility fractures and osteonecrosis of the jaw in patients on bisphosphonates have been described but remain relatively rare. It is plausible that atypical fragility fractures could follow from suppression of bone remodelling, but high-quality studies proving causality are lacking. A recent secondary analysis of 3 large bisphosphonate trials found no significant increase in the risk of atypical fracture associated with bisphosphonate use for up to 10 years. ${ }^{[34]}$ However the study was not adequately powered for definitive conclusions. A further British cohort study using national healthcare data found alendronate-treated patients to be at higher risk of hip and subtrochanteric/diaphyseal fracture than matched control subjects. ${ }^{[35]}$ Large cumulative doses of alendronate were not however associated with a greater absolute risk of subtrochanteric/diaphyseal fractures than small cumulative doses, suggesting that these fractures could be due to osteoporosis rather than to alendronate.

Osteonecrosis of the jaw (ONJ) has been described in patients receiving bisphosphonate therapy and to date several aetiological theories have been proposed including inhibition of bone remodelling, effect on soft tissues, and antiangiogenic effect of bisphosphonates. However almost all cases to date have been described in cancer patients on high dose intravenous formulations and are often 
associated with dental procedures. The European Medicines Agency in a recent review concluded that the risk of ONJ is higher in those patients receiving intravenous bisphosphonates for cancer than for patients receiving oral therapy. ${ }^{[36]}$

\section{Hormone replacement therapy}

Numerous randomised placebo-controlled studies including the Womens health initiative (WHI) ${ }^{[37]}$ and the postmenopausal oestrogen/progesterone interventions (PEPI) ${ }^{[38]}$ have established that the postmenopausal decrease in BMD is attenuated by oestrogen, resulting in a lower risk of fracture. However data from the WHI also suggested that oestrogen/progesterone therapy increases the incidence of breast cancer, coronary heart disease, stroke and venous thromboembolism. Unopposed oestrogen therapy has been shown to increase stroke and thromboembolism only. Therefore as overall health risks exceed benefits, hormone replacement is not considered first-line therapy in the management of postmenopausal osteoporosis.

\section{Selective Estrogen Receptor Modulators}

Raloxifene, lasofoxifene and bazedoxifene are currently the SERMs available for use in the treatment of osteoporosis and their effects on vertebral and nonvertebral fractures have been studied in numerous trials reporting beneficial results. The first SERM used to treat osteoporosis, raloxifene, demonstrated a reduced risk of vertebral fractures (HR 0.7) but not nonvertebral fracture in a large 3

year study of postmenopausal women. ${ }^{[39]}$ However, subsequent post hoc analyses reported a decrease in new vertebral and nonvertebral fracture risk in the subgroup of women with severe vertebral fractures at baseline. ${ }^{[40]}$ Bazedoxifene significantly reduced the risk of new vertebral fracture in postmenopausal women with osteoporosis and decreased the risk of nonvertebral fracture in subjects at higher fracture risk ${ }^{[41]}$ and in the recent PEARL study of postmenopausal women with osteoporosis, lasofoxifene was associated with reduced risk of both nonvertebral and vertebral fractures. ${ }^{[42]}$ Although SERMs have demonstrated benefits with regard to ER-positive breast cancer, coronary heart disease, and stroke, the main concern with treatment is the three-fold increased risk of venous thromboembolism. Ospemifene and arzoxifene are new SERM molecules currently under investigation. ${ }^{[43,44]}$

\section{Anabolic therapy}




\section{Parathyroid Hormone}

Recombinant Parathyroid Hormone (PTH) 1-34 and 1-84 are anabolic agents which work by stimulating preosteoblasts to mature into bone-forming osteoblasts. When given at low dose by daily subcutaneous injection they enhance bone remodelling. Bone formation begins within the first month of treatment and bone resorption begins after 6 months. ${ }^{[45]}$ Therefore during the first year of treatment bone remodelling is in a positive balance. In the Fracture Prevention Trial, postmenopausal women with previous vertebral fracture were randomised to PTH (20 or $40 \mathrm{mcg}$ ) or placebo. BMD in the $20 \mathrm{mcg}$ and $40 \mathrm{mcg}$ groups increased both at the lumbar spine ( $9 \%$ and $13 \%$ respectively) and at the femoral neck (3\% and $6 \%$ respectively). However overall fracture risk reduction was proportionately greater and found to be very similar for both dosage groups. In the $20 \mathrm{mcg}$ group, where side effects were less common, the respective relative risks of vertebral and non-vertebral fractures were 0.35 and 0.47. Subgroup analysis showed that the benefits were independent of age, baseline BMD and prevalent vertebral fractures.

The changes in BMD seen with PTH are early and of greater magnitude than those seen with other treatments and it therefore has a role in patients with severe established disease who are at particularly high risk for fragility fractures; $T$ score $\leq-3.5$ without fracture or $T$ score $\leq-2.5$ with fragility fracture. However the strongest evidence to date in support of the efficacy of PTH in reducing vertebral and non-vertebral fractures showed that its use is likely to be most effective in patients with pre-existing fragility fractures and not necessarily associated with very low BMD score. ${ }^{[46]}$

The main concern with long term treatment is development of osteosarcoma as this was seen in rat models with high dose treatment. ${ }^{[47]}$ Fortunately in practice this has not been observed and very few long term side effects have been reported. Due to concern regarding long term safety and plateau in bone formation with treatment beyond 18 months, treatment is limited to low dose for a maximum of 2 years. PTH is not usually considered first-line therapy due to cost, route of administration and paucity of strong data on nonvertebral fracture reduction. It has a role however in patients with severe disease and when other therapies are not tolerated or have failed. Anti-resorptives should however be reintroduced following discontinuation of PTH to preserve the BMD gained on treatment, as demonstrated in an extension of the PaTH study. ${ }^{[48]}$

\section{Dual action - anti-resorptive and anabolic}




\section{Strontium ranelate}

Strontium ranelate has a unique mode of action: it dissociates bone remodelling by reducing bone resorption while allowing continued bone formation. Its effect on vertebral fractures was assessed in SOTI, a trial in which postmenopausal women received daily strontium ranelate or placebo for three years. ${ }^{[49]}$ A relative risk reduction in vertebral fractures was seen in favour of strontium; $49 \%$ after 1 year and $41 \%$ over 3 years (RR 0.59 ). Strontium ranelate increased bone mineral density at month 36 by $14.4 \%$ at the lumbar spine and $8.3 \%$ at the femoral neck ( $P<0.001$ for both). Efficacy was subsequently assessed in prevention of non-vertebral fractures in TROPOS, a double-blind 5-year study of 5091 postmenopausal women. ${ }^{[50]}$ Relative risk for major fragility fractures was reduced in the strontium group by $19 \%$ compared with the placebo group. On extension of these studies to 8 years, BMD of lumbar spine, femoral neck and total hip continued to increase with some evidence for sustained anti-fracture efficacy and strontium was found to be safe and well tolerated. ${ }^{[51]}$

Women $\geq 80$ years are at higher relative risk of vertebral and non-vertebral fractures and subgroup analysis of these studies concluded an early and sustained reduction of both vertebral and nonvertebral fractures with strontium in these patients. ${ }^{[52]}$ Caution has been advised when interpreting BMD values when on strontium as a proportion of the change in value may be due to accumulation of strontium in the bone tissue however an increase in femoral neck BMD has been shown to be associated with a proportional reduction in vertebral fracture incidence. ${ }^{[53]}$ Side effects include transient nausea, diarrhoea and headache. A slight excess of venous thromboembolism (VTE) in patients with a history of thromboembolism has been noted in some but not all trials. A recent large retrospective cohort study reported a greater association of VTE in osteoporotic compared with nonosteoporotic patients (RR 1.75; $\mathrm{Cl} 1.09$ to 1.84 ) but no increased incidence in those treated with strontium compared with alendronate. ${ }^{[54]}$

\section{Novel therapeutic Agents for the management of osteoporosis}

\section{Denosumab}


Denosumab (AMG 162) is a human monoclonal antibody with a high affinity and specificity for RANKL, a key mediator of osteoclastic bone resorption. By reducing RANKL binding to the osteoclast receptor RANK, there is a decrease in differentiation, activity, and survival of osteoclasts, lowering the rate of bone resorption. ${ }^{[55]}$ In a dose-ranging study the efficacy and safety of subcutaneously administered denosumab were evaluated in 412 postmenopausal women (mean age 62 years) with low BMD. ${ }^{[56]}$ Patients were randomised to receive denosumab or alendronate. Denosumab resulted in an increase in BMD from baseline at the lumbar spine of up to 6.7 percent (vs $4.6 \%$ with alendronate) and at the total hip of up to 3.6 percent (vs $2.1 \%$ with alendronate). This study was extended to 24 months and a sustained increase in BMD at total hip, lumbar spine and distal radius was recorded with no increase in adverse events. ${ }^{[57]}$ The effects on bone turnover were found to be fully reversible on discontinuation of the drug but restored with subsequent treatment. ${ }^{[58]}$ A further larger study of 1 189 postmenopausal women randomised to denosumab $60 \mathrm{mg} 6$ monthly or alendronate $70 \mathrm{mg}$ weekly showed significant increase in BMD at all measured skeletal sites in patients on denosumab at 12 months $(3.5 \%$ vs $2.6 \%, p<0.0001)$. There was a significantly greater reduction of bone turnover markers and the safety profile was similar for both treatments. ${ }^{[59]}$

The FREEDOM trial of 7868 women aged 60 to 90 years with osteoporosis (BMD T score between 2.5 and -4.0$)$ at the lumbar spine or total hip showed very promising results. ${ }^{[60]}$ Subjects were assigned to receive either $60 \mathrm{mg}$ of denosumab or placebo subcutaneously every 6 months for 36 months. Denosumab reduced the risk of new radiographic vertebral fracture by $68 \%$ and reduced the risk of hip fracture by $40 \%$. It also reduced the risk of nonvertebral fracture by $20 \%$. Adverse events were similar to placebo indicating a favourable safety profile. In this large study there was no increase in the risk of cancer, infection, cardiovascular disease, delayed fracture healing or hypocalcemia and no adverse reactions to the injection of denosumab. Since the publication of this study, suspected cases of ONJ have been described in patients on denosumab ${ }^{[61,62]}$ but to date these case reports remain very limited. Significantly greater treatment adherence was observed for subcutaneous administration of denosumab every 6 months than for oral alendronate once weekly ${ }^{[63]}$ with a risk ratio for non-adherence at 12 months of $0.58(p=0.043)$ for denosumab compared with alendronate. Patient preference and satisfaction were also significantly greater for denosumab. The FDA (Food and 
drug administration) and EMEA (European Medicines Agency) have approved denosumab for the treatment of postmenopausal osteoporosis and it is currently available for use in clinical practice.

\section{Odanacatib}

Cathepsin $\mathrm{K}$ is a lysosomal cysteine protease expressed in abundance by osteoclasts which degrades Type I collagen and is responsible for degradation of the organic bone matrix during bone remodelling. Odanacatib (MK-822) is a potent and selective inhibitor of Cathepsin $\mathrm{K}^{[64]}$ and has been shown in studies to reduce osteoclast-mediated bone resorption. In two double-blind, randomized, placebocontrolled studies, postmenopausal females received odanacatib $2.5 \mathrm{mg}$ once weekly for 3 weeks or once daily for 21 days. It was well tolerated and had a long half-life $\left(t_{1 / 2} 66-93 \mathrm{~h}\right)$ consistent with onceweekly dosing. A reduction in biochemical markers of bone resorption of up to $81 \%$ was seen ${ }^{[65]} \mathrm{A}$ recent study of 399 women with BMD T-scores between -2.0 and -3.5 at the lumbar spine or femoral neck was performed whereby participants were randomly assigned to receive placebo or one of four doses of odanacatib $(3,10,25$ or $50 \mathrm{mg}$ weekly) over 24 months. After 24 months, lumbar spine and total hip BMD increased 5.5\% and 3.2\% respectively in patients on $50-\mathrm{mg}$ odanacatib compared with placebo. ${ }^{[66]}$ Unlike other less selective cathepsin inhibitors which caused skin reactions, the safety and tolerability of odanacatib are similar to placebo. Results of ongoing large scale trials to evaluate the effects of odanacatib on fracture risk in postmenopausal women are awaited.

\section{Glucagon-like peptide 2 (GLP-2)}

There is a circadian pattern to bone remodelling, as shown by biochemical assessments, whereby bone resorption is reduced during the daytime and increased at night, and this pattern has been linked to food intake. GLP-2 is released in the small intestine almost immediately after food intake and its secretion is therefore reduced overnight with nocturnal fasting. The presence of GLP-2 receptors in osteoclasts suggests that GLP-2 may form part of an 'entero-osseous axis', coordinating nutrientdependent bone turnover. As bone resorption occurs at increased rate during this fasting period it was hypothesised that administration of GLP-2 at bedtime may reduce this rate of bone resorption overnight. A double-blind placebo-controlled trial of 60 postmenopausal women administered GLP-2 daily for 14 days showed a significant and sustained reduction in bone resorption (Ctx) with the added advantage of not decreasing bone formation (OC), unlike other antiresorptive agents ${ }^{[67]}$ The investigators then extended the study period to four months. This phase II, placebo-controlled trial of 
160 postmenopausal women treated with GLP-2 ( 3 doses) over 120 days similarly resulted in a reduction in nocturnal rise of C-telopeptide and no effect on osteocalcin and an increase in hip BMD $(1.1 \%$ with highest dose of $3.2 \mathrm{mg}, \mathrm{p}=0.007) .{ }^{[68]}$ The overall rates of adverse events in the treatment groups were similar and there were no signs of tachyphlaxis or antibody production against GLP2.The results indicate that GLP-2 produces a substantial decrease in bone resorption without suppression of bone formation thereby changing the bone remodelling balance in favour of bone formation, particularly at the hip. If this disassociation between bone resorption and formation can be sustained with no adverse effects, it may prove an interesting future therapy for osteoporosis. Studies are also currently underway looking at GLP-1 as a possible anabolic agent to improve deficiencies in bone formation associated with glucose intolerance.

\section{Wnt signalling: Inhibitors of Sclerostin and Dickkopf 1(Dkk-1)}

Wnt proteins are a large family of extracellular glycoproteins involved in many cellular processes. The Wnt/beta-catenin signaling pathway has emerged as a key regulator of bone homeostasis and recently neutralizing inhibitors have emerged as a possible therapeutic option for the management of osteoporosis. $^{[69]}$ These include antibodies to Sclerostin and Dkk-1 and also inhibitors of secreted Frizzled-related protein 1 and glycogen synthase kinase 3beta. Sclerostin is secreted by osteocytes and genetic studies have shown that it is a key negative regulator of bone formation. Its inhibition was assessed using a cell culture model of bone formation to identify a sclerostin neutralizing monoclonal antibody (Scl-Abll). ${ }^{[70]}$ When ovariectomized rats were administered Scl-Abll for 5 weeks robust anabolic effects were noted on trabecular, periosteal, endocortical, and intracortical surfaces. It resulted in complete reversal of bone loss at several skeletal sites but also further increased bone mass and bone strength to levels greater than those found in control rats. In the first human study a single subcutaneous dose of Scl-Abll (AMG 785) was administered to 72 healthy men and postmenopausal women. It resulted in an increase in P1NP and osteocalcin and a dose related decrease in the bone marker CTx at day 84 of treatment. An increase of $5.3 \%$ in BMD was seen at the lumbar spine and of $2.8 \%$ at the total hip. Neutralising antibodies developed in 2 patients but had no effect on the pharmacokinetics of this agent. ${ }^{[71]}$ Dkk-1 is a negative regulator of bone homeostasis and levels are significantly higher in osteoporotic women compared with controls. ${ }^{[72]}$ Manipulation of its function and expression has therapeutic significance for the treatment of osteoporosis and progressive 
Dkk-1 reduction has been shown to increase trabecular and cortical bone mass in mice and primates. ${ }^{[73,74]}$ Human studies showing beneficial effects are currently underway.

\section{Calcium Sensing Receptor, Activin Receptor Fusion Protein \& Integrin aub3}

Potential future targets for osteoporosis include calcium sensing receptor, activin receptor fusion protein and integrin aub3. Antagonists to calcium sensing receptor result in transient PTH surge with each oral dose similar to that seen with intermittently administered exogenous PTH. Activin, a cofactor for osteoclastogenesis, binds to activin receptor IIA and an antibody to it has shown a reduction Ctelopeptide in postmenopausal women. Antibodies to alpha(v)beta(3) integrin, expressed on osteoclasts, inhibit bone resorption in vitro and in vivo, suggesting an important role in regulating osteoclast function. Although interesting novel targets, future studies will determine long-term tolerability and safety. It is likely to be some years before these agents are introduced to clinical practice.

\section{Prescribing Considerations}

Osteoporosis is predominantly a disease of older adults so, when prescribing, other factors such as cognition, compliance, co-morbidities and route of administration must be taken into consideration. Medication effectiveness needs to be balanced against tolerability in individual patients. All patients should be on calcium supplementation and should be vitamin D replete. For the well, compliant older person, with no contra-indications, oral weekly bisphosphonate therapy or oral daily strontium are considered first line. However hip fracture is the main concern amongst older patients with osteoporosis and bisphosphonates have well documented hip fracture risk reduction which is maintained over many years.

Repletion of Vitamin D is particularly important in the frailest older patients, such as nursing home residents or older patients with poor mobility and limited sunlight exposure. It may be given intramuscularly if compliance is uncertain. ${ }^{[75]}$ It also suppresses parathyroid hormone, increases bone mineral density and reduces falls ${ }^{[76]}$ and hip fracture.

In patients who have difficulty swallowing medications or in those intolerant of oral bisphosphonates due to upper gastrointestinal disturbance, annual intravenous zolendronic acid or subcutaneous 
denosumab are useful options. It should also be considered when compliance with oral therapy remains an issue in community-dwelling patients.

More than half of all fractures in the community occur in patients with osteopenia. However most studies assessing antifracture efficacy of drugs include osteoporotic patients who are at higher absolute risk for fracture. In cases of osteopenia, treatment with strontium can be considered. Pooled data from SOTI and TROPOS reported significant antifracture efficacy of strontium ranelate in women with lumbar spine (LS) and femoral neck (FN) osteopenia. ${ }^{[77]}$ The risk of clinical vertebral fractures was reduced by $60 \%$ with alendronate in a subgroup of patients from the FIT 1 study with FN osteopenia and prevalent fractures ${ }^{[78]}$ Further studies are needed to determine effective therapies to reduce hip fracture risk in patients with osteopenia.

PTH is recommended as alternative treatment to bisphosphonates or strontium in the setting of a contraindication, intolerance to or failure of these drugs. Despite PTH's known efficacy, due to its high cost and without very strong evidence to support superiority to other treatments in antifracture efficacy, its use is likely to remain relatively restricted.

\section{Prescribing in patients with reduced GFR}

Osteoporosis in older persons often co-exists with chronic kidney disease (CKD) and could be included in the broad spectrum of metabolic bone conditions that accompany a decline in renal function. As the population prevalence of CKD is increasing, the management of osteoporosis in these patients is a frequently encountered problem. In advanced stages of CKD it is important to discriminate between osteoporosis and other forms of renal osteodystrophy, which can also impair bone strength and lead to fracture. WHO criteria for diagnosing osteoporosis can be applied in CKD stages 1 to 3 , once biochemical profiling is normal. In these cases current registered therapies for osteoporosis have proven safety and can be used. In the setting of fragility fractures in more advanced stages of kidney disease the diagnosis and management of osteoporosis is less clear. Careful assessment of bone turnover using serum levels of parathyroid hormone and bone-specific alkaline phosphatase is needed. In some cases, double-tetracycline labelled bone biopsy may be indicated to outrule adynamic bone disease before considering anti-resorptive treatment. In fracture patients with osteoporosis and stages 4 and 5 CKD treatment is difficult as therapies remain 
unlicenced in this setting. However post hoc analyses of trials using risedronate, alendronate and raloxifene have reported tolerability of these drugs in stage $4 \mathrm{CKD} \cdot{ }^{[79-81]}$ No efficacy data however supports their use in end stage kidney disease. If recurrent fragility fractures pose a great risk to these patients it may be reasonable, based on known pharmacokinetics, to reduce the dose of bisphosphonate by half after clear diagnosis is made and consent from the patient is sought. In patients with renal osteodystrophy on dialysis, ibandronate has shown significant increase in BMD and decrease in $\mathrm{BTM}^{\left[{ }^{[82]}\right.}$ Alendronate has also shown a bone preserving effect in patients on haemodialysis. ${ }^{[83]}$ In all patients with advanced CKD supplementation with active vitamin D is essential to reduce the effects of secondary hyperparathyroidism. In summary no consensus exists to guide our treatment decisions in cases of osteoporosis in stages 4 and 5 CKD and further large-scale prospective studies are needed. As denosumab is a monoclonal antibody, it is not cleared by the kidneys, which makes it a safer option for people who have renal disease. In patients with varying degrees of renal function, including patients on dialysis, the degree of renal impairment had no effect on the pharmacokinetics of denosumab. Therefore no dose adjustments of this drug are required with different degrees of renal impairment.

\section{Conclusion.}

The incidence of osteoporosis-related fractures will rise substantially over the coming decades as the population ages. This increased incidence will have significant social and economic implications for most countries, particularly in the developed world. In recent years our understanding of normal bone physiology and the mechanisms underlying development of osteoporosis has significantly advanced and this has led to the development of new therapies. Currently we have good anti-fracture data to support the use of many available anti-resorptive and anabolic drugs and supplementation of calcium and vitamin D. Newer agents, particularly denosumab, will increase the therapeutic options for clinicians in the coming years. Determination of future fracture risk, evaluation of co-morbidities, measurement of renal function and estimation of potential compliance difficulties are all important considerations for the prescribing physician to take into account when deciding upon which agents to use in individual patients.

\section{$\underline{\text { References }}$}


Sambrook P, Cooper C. Osteoporosis. Lancet 2006;367:2010-18.

2.

Cummings SR, Melton LJ. Epidemiology and outcomes of osteoporotic fractures. Lancet 2002;359:1761

3

Heaney RP. Remodelling and skeletal fragility. Osteoporos Int 2003;14(suppl 5):S12-15

4

Bruyere O, Varela AR, Ademi S et al. Loss of hip bone mineral density over time in association with spine and hip fracture incidence in osteoporotic postmenopausal women. Eur $\mathrm{J}$ Epidemiol. 2009;24(11):707-12.

\section{5}

Seibel MJ, Naganathan V, Barton I et al. Relationship between pretreatment bone resorption and vertebral fracture incidence in postmenopausal osteoporotic women treated with risedronate. $J$ Bone Miner Res 2004;19:323

6

Bauer DC, Black DM, Garnero P et al. Change in bone turnover and hip, non-spine and vertebral fracture in alendronate-treated women: the fracture intervention trial. J Bone Miner Res 2004;19:1250

7

Bauer DC, Garnero P, Hochberg MC et al. Pretreatment levels of bone turnover and the antifracture efficacy of alendronate: the fracture intervention trial. J Bone Miner Res 2006;21:292

8

Cummings SR, Nevitt MC, Browner WS et al. Risk factors for hip fracture in white women. N Engl J Med 1995;332:767.

9

Kanis $\mathrm{J}$ et al. FRAX and the assessment of fracture probability in men and women from the UK. Osteoporosis Int 2008; 19:385.

10

Kanis JA, McCloskey EV, Johansson $\mathrm{H}$ et al. Case finding for the management of osteoporosis with FRAX--assessment and intervention thresholds for the UK. National Osteoporosis Guideline Group. 2008 Oct;19(10):1395-408.

11

Borgström F, Ström O, Coelho $\mathrm{J}$ et al. The cost-effectiveness of risedronate in the UK for the management of osteoporosis using the FRAX. Osteoporos Int. 2010 Mar;21(3):495-505. Epub 2009 Jun 30

12 
Borgström F, Ström O, Coelho $\mathrm{J}$ et al. The cost-effectiveness of strontium ranelate in the UK for the management of osteoporosis. Osteoporos Int. 2010 Feb;21(2):339-49. Epub 2009 Jun 10

13

Hippisley-Cox J, Coupland C. Predicating risk of osteoporotic fracture in men and women in England and Wales: prospective derivation and validation of QFractureScores. BMJ. 2009;339:b4229.

Holick MF. Optimal vitamin D status for the prevention and treatment of osteoporosis. Drugs Aging. 2007;24(12):1017-29.

15

Bischoff-Ferrari, HA, Dietrich, T, Orav, E et al. Positive association between 25-hydroxy vitamin D levels and bone mineral density: a population-based study of younger and older adults. Am J Med 2004;116:634.

16

Jackson RD, LaCroix AZ, Gass M, et al. Calcium plus vitamin D supplementation and the risk of fractures. N Engl J Med 2006;354:669

17

Dawson-Hughes B, Harris SS, Krall EA et al.. Effect of calcium and vitamin D supplementation on bone density in men and women 65 years of age or older. N Engl J med 1997;337:670

18

Prince RL, Devine A, Dhaliwal SS et al. Effects of Calcium Supplementation on Clinical Fracture and Bone Structure. Results of a 5-Year, Double-blind, Placebo-Controlled Trial in Elderly Women. Arch Intern Med. 2006;166:869-875.

19

Dawson-Hughes B, Bischoff-Ferrari HA.Jean Mayer. Therapy of osteoporosis with calcium and vitamin D. J Bone Miner Res. 2007 Dec;22 Suppl 2:V59-6.

20

Bolland MJ, Avenell A, Baron J et al. Effect of calcium supplements on risk of myocardial infarction and cardiovascular events: meta-analysis. BMJ 2010;341:c3691.

21

Dunford JE. Molecular Targets of the Nitrogen Containing Bisphosphonates: - The Molecular Pharmacology of Prenyl Synthase Inhibition. Curr Pharm Des. 2010 Aug 20. [Epub ahead of print]

22

Black DM, Cummings SR, Karpf DB et al. Randomised trial of effect of alendronate on risk of fracture in women with existing vertebral fractures. Fracture Intervention Trial Research Group. Lancet. 1996 Dec 7;348(9041):1535-41. 
Cummings SR, Black DM, Thompson DE, et al. Effect of alendronate on risk of fracture in women with low bone density but without vertebral fractures. Results from the Fracture Intervention Trial. JAMA 1998; 280:2077.

Harris ST, Watts NB, Genant HK et al. Effects of risedronate treatment on vertebral and non-vertebral fractures in women with postmenopausal osteoporosis: a randomized controlled trial. Vertebral Efficacy With Risedronate Therapy (VERT) Study Group. JAMA 1999; 282:1344.

25

McClung MR, Geusens P, Miller PD et al. Effect of risedronate on the risk of hip fracture in elderly women. N Engl J Med 2001;344:333

\section{6}

Delmas PD, Recker RR, Chesnut $\mathrm{CH}$ 3rd et al. Daily and intermittent oral ibandronate normalize bone turnover and provide significant reduction in vertebral fracture risk: results from the BONE study. Osteoporos Int. 2004 Oct;15(10):792-8.

\section{7}

Harris ST, Reginster JY, Harley C et al. Risk of fracture in women treated with monthly oral ibandronate or weekly bisphosphonates: the eValuation of IBandronate Efficacy (VIBE) database fracture study. Bone. 2009 May;44(5):758-65.

\section{8}

Black DM, Delmas PD, Eastell R et al. Once-yearly zolendronic acid for treatment of postmenopausal osteoporosis. N Engl J Med 2007; 356:1809

\section{9}

Lyles KW, Colon-Emeric CS, Magaziner JS et al. Zolendronic acid and clinical fractures and mortality after hip fracture. N Engl J Med 2007; 357:1799

30

Eriksen EF, Lyles KW, Colón-Emeric CS et al. Antifracture efficacy and reduction of mortality in relation to timing of the first dose of zoledronic acid after hip fracture. Bone Miner Res. 2009 Jul;24(7):1308-13.

\section{1}

Wysowski DK. Reports of esophageal cancer with oral bisphosphonate use. N Engl J Med. 2009 Jan 1;360(1):89-90.

\section{2}

Cardwell CR, Abnet CC, Cantwell MM et al. Exposure to Oral Bisphosphonates and risk of Esophageal cancer. JAMA 2010 Aug 11;304(6):657-663

\section{3}

Green J, Czanner G, Reeves $\mathrm{G}$ et al. Oral bisphosphonates and risk of cancer of oesophagus, stomach, and colorectum: case-control analysis within a UK primary care cohort. BMJ. 2010 Sep $1 ; 341: c 4444$. 
Black DM, Kelly MP, Genant HK et al. Bisphosphonates and Fractures of the Subtrochanteric or Diaphyseal Femur. N Engl J Med. 2010 May 13;363(19):1761-71.

\section{5}

Abrahamsen B, Eiken P, Eastell R. Cumulative Alendronate Dose and the Long-Term Absolute Risk of Subtrochanteric and Diaphyseal Femur Fractures: A Register-Based National Cohort Analysis. J Clin Endocrinol Metab. 2010 Sep 15. [Epub ahead of print]

36

CHMP assessment report on bisphosphonate and osteonecrosis of the jaw (2009). www.emea.europa.eu

\section{7}

Rossouw JE; Anderson GL; Prentice RL et al. Risks and benefits of estrogen plus progestin in healthy postmenopausal women: principal results From the Women's Health Initiative randomized controlled trial. JAMA 2002 Jul 17;288(3):321-33

38

Effects of hormone therapy on bone mineral density: results from the postmenopausal estrogen/progestin interventions (PEPI) trial. The Writing Group for the PEPI. JAMA 1996 Nov 6;276(17):1389-96.

\section{9}

Ettinger B, Black DM, Mitlak BH et al. Reduction of vertebral fracture risk in postmenopausal women with osteoporosis treated with raloxifene: results from a 3-year randomized clinical trial. Multiple Outcomes of Raloxifene Evaluation (MORE) Investigators. JAMA. 1999 Aug 18;282(7):637-45.

40

Delmas PD, Genant HK, Crans GG et al. Severity of prevalent vertebral fractures and the risk of subsequent vertebral and nonvertebral fractures: results from the MORE trial. Bone. 2003 Oct;33(4):522-32.

\section{1}

Silverman SL, Christiansen C, Genant HK et al. Efficacy of bazedoxifene in reducing new vertebral fracture risk in postmenopausal women with osteoporosis: results from a 3-year, randomized, placebo-, and active-controlled clinical trial. J Bone Miner Res. 2008 Dec;23(12):1923-34.

42

Cummings SR, Ensrud K, Delmas PD et al. PEARL Study Investigators. Lasofoxifene in postmenopausal women with osteoporosis. N Engl J Med 2010 Feb 25;362(8):686-96.

43

Komi J, Lankinen KS, DeGregorio $\mathrm{M}$ et al. Effects of ospemifene and raloxifene on biochemical markers of bone turnover in postmenopausal women. J Bone Miner Metab. 2006;24(4):314-8.

44 
Bolognese $\mathrm{M}$, Krege $\mathrm{JH}$, Utian $\mathrm{WH}$ et al. Effects of arzoxifene on bone mineral density and endometrium in postmenopausal women with normal or low bone mass. J Clin Endocrinol Metab. $2009 \mathrm{Jul} ; 94(7): 2284-9$.

45

Black DM, Greenspan SL, Ensrud KE et al. The effects of parathyroid hormone and alendronate alone or in combination in postmenopausal osteoporosis. N Engl J Med 2003; 349:1207.

\section{6}

Neer RM, Arnaud CD, Zanchetta JR et al. Effect of Parathyroid Hormone (1-34) on Fractures and Bone Mineral Density in Postmenopausal Women with Osteoporosis. N Engl J Med 2001; 344:1434.

\section{7}

Vahle JL, Sato M, Long GG et al. Skeletal changes in rats given daily subcutaneous injections of recombinant human parathyroid hormone (1-34) for 2 years and relevance to human safety. Toxicol Pathol 2002;30:312

48

Black DM, Bilezikian JP, Ensrud KE et al. One year of alendronate after one year of parathyroid hormone (PTH 1-84) for osteoporosis. N Engl J Med 2005; 353:555

\section{9}

Meunier PJ, Roux C, Seeman E et al. The effects of strontium ranelate on the risk of vertebral fracture in women with postmenopausal osteoporosis (SOTI). N Engl J Med. 2004 Jan 29;350(5)

50

Seeman E, De Vernejoul MC, Adami $S$ et al. Strontium ranelate reduces the risk of nonvertebral fractures in postmenopausal women with osteoporosis: Treatment of Peripheral Osteoporosis (TROPOS) study. J Clin Endocrinol Metab. 2005 May;90(5):2816-22.

\section{1}

Reginster JY, Bruyère $O$, Sawicki $A$ et al. Long-term treatment of postmenopausal osteoporosis with strontium ranelate: results at 8 years. Bone. 2009 Dec;45(6):1059-64.

\section{2}

Seeman E, Vellas B, Benhamou C et al. Strontium ranelate reduces the risk of vertebral and nonvertebral fractures in women eighty years of age and older. $J$ Bone Miner Res. 2006 Jul;21(7):1113-20.

\section{3}

Bruyere O, Roux C, Detilleux $\mathrm{J}$ et al. Relationship between bone mineral density changes and fracture risk reduction in patients treated with strontium ranelate. J Clin Endocrinol Metab. 2007 Aug;92(8):3076-81.

\section{4}

Breart G, Cooper C, Meyer O et al. Osteoporosis and venous thromboembolism: a retrospective cohort study in the UK General Practice Research Database. Osteoporos Int. 2010 Jul;21(7):1181-7. 
Lewiecki EM. Denosumab: an investigational drug for the management of postmenopausal osteoporosis. Biologics. 2008 Dec;2(4):645-53

56

McClung MR, Lewiecki EM, Cohen SB et al. AMG 162 Bone Loss Study Group. Denosumab in postmenopausal women with low bone mineral density. N Engl J Med. 2006 Feb 23;354(8):821-31.

\section{7}

Lewiecki EM, Miller PD, McClung MR et al. Two year treatment with denosumab (AMG 162) in a randomized phase 2 study of postmenopausal women with low BMD. J Bone Miner Res. 2007 Dec;22(12):1832-41.

58

Miller PD, Bolognese MA, Lewiecki EM et al. Effect of denosumab on bone density and turnover in postmenopausal women with low bone mass after long-term continued, discontinued, and restarting of therapy: a randomised blinded phase 2 clinical trial. Bone 2008 Aug;43(2):222-9.

\section{9}

Brown JP, Prince RL, Deal $C$ et al. Comparison of the effect of denosumab and alendronate on BMD and biochemical markers of bone turnover in postmenopausal women with low bone mass: a randomized, blinded, phase 3 trial. J Bone Miner Res. 2009 Jan;24(1):153-61.

60

Cummings SR, San Martin J, McClung MR et al. FREEDOM Trial. Denosumab for prevention of fractures in postmenopausal women with osteoporosis. N Engl J Med. 2009 Aug 20;361(8):756-65.

61

Aghaloo T, Felsenfeld A, Tetradis S. Osteonecrosis of the Jaw in a Patient on Denosumab. Journal of Oral and Maxillofacial Surgery. Volume 68, Issue 5, May 2010, Pages 959-963.

62

Taylor KH, Middlefell LS, Mizen KD. Osteonecrosis of the jaws induced by anti-RANK ligand therapy. Br J Oral Maxillofac Surg. 2010 Apr;48(3):221-3.

63

Kendler DL, McClung MR, Freemantle $\mathrm{N}$ et al on behalf of the DAPS Investigators. Adherence, preference, and satisfaction of postmenopausal women taking denosumab or alendronate. 2010 Sep 9. [Epub ahead of print]

64

Gauthier JY, Chauret N, Cromlish W et al. The discovery of odanacatib (MK-0822), a selective inhibitor of cathepsin K. Bioorganic \& Medicinal Chemistry Letters. Volume 18, Issue 3, 1 February 2008:923-928.

\section{5}

Stoch SA, Zajic S, Stone J et al. Effect of the cathepsin K inhibitor odanacatib on bone resorption biomarkers in healthy postmenopausal women: two double-blind, randomized, placebo-controlled phase I studies. Clin Pharmacol Ther. 2009 Aug;86(2):175-82. 
Bone HG, McClung MR, Roux C et al. Odanacatib, a Cathepsin-K Inhibitor for Osteoporosis: A TwoYear Study in Postmenopausal Women With Low Bone Density. J Bone Miner Res. 2010 may;25(5):937-47

\section{7}

Henriksen DB, Alexandersen $P$, Hartmann $B$ et al. Disassociation of bone resorption and formation by GLP-2. A 14 day study in healthy postmenopausal women. Bone 2007 Mar 40(3):723

68

Henriksen DB, Alexandersen P, Hartmann B et al. Four month treatment with GLP-2 significantly increases hip BMD: a randomized, placebo-controlled, dose-ranging study in postmenopausal women with low BMD. Bone 2009 Nov;45(5):833-42.

69

Hoeppner LH, Secreto FJ, Westendorf JJ. Wnt signaling as a therapeutic target for bone diseases. Expert Opin Ther Targets. 2009 Apr;13(4):485-96.

70

Li X, Ominsky MS, Warmington KS et al. Sclerostin antibody treatment increases bone formation, bone mass, and bone strength in a rat model of postmenopausal osteoporosis. J Bone Miner Res. 2009 Apr;24(4):578-88.

Padhi D, Jang G, Stouch B, Fang $L$ et al. Single-dose, placebo-controlled, randomised study of AMG 785, a sclerostin monoclonal antibody. J Bone Miner Res. 2010; Jun 30 [Epub ahead of print].

72

Anastasilakis AD, Polyzos SA, Avramidis A.. The effect of teriparatide on serum Dickkopf-1 levels in postmenopausal women with established osteoporosis. Clin Endocrinol (Oxf). 2009 Oct 15.

73

MacDonald BT, Joiner DM, Oyserman SM et al. Bone mass is inversely proportional to Dkk1 levels in mice. Bone. 2007 Sep;41(3):331-9.

\section{4}

Betts AM, Clark TH, Yang $J$ et al. The application of target information and preclinical pharmacokinetic/ pharmacodynamic modeling in predicting clinical doses of a Dickkopf-1 antibody for osteoporosis. J Pharmacol Exp Ther. 2010 Apr;333(1):2-13.

75

Nugent C, Roche K, Wilson S et al. The effect of intramuscular vitamin D (cholecalciferol) on serum $25 \mathrm{OH}$ vitamin D levels in older female acute hospital admissions. Ir J Med Sci. 2009 Aug 28. [Epub ahead of print]

\section{6}

Harwood RH, Sahota O, Gaynor K et al. Nottingham Neck of Femur (NONOF) Study. A randomised, controlled comparison of different calcium and vitamin $\mathrm{D}$ supplementation regimens in elderly women 
after hip fracture: The Nottingham Neck of Femur (NONOF) Study. Age Ageing. 2004 Jan;33(1):4551.

77

Seeman E, Devogelaer JP, Lorenc $R$ et al. Strontium ranelate reduces the risk of vertebral fractures in patients with osteopenia. J Bone Miner Res 2008;23:433-8.

78

Quandt SA, Thompson DE, Schneider DL et al. Effect of alendronate on vertebral fracture risk in women with bone mineral density $T$ scores of -1.6 to -2.5 at the femoral neck. Mayo Clin Proc.2005;80:343-9.

79

Miller PD, Roux C, Boonen S et al. Safety and efficacy of risedronate in patients with age-related reduced renal function as estimated by the Cockcroft and Gault method: a pooled analysis of nine clinical trials. J Bone Miner Res 2005;20:2105-15.

80

Jamal SA, Bauer DC, Ensrud et al. Alendronate treatment in women with normal to severely impaired renal function: an analysis of the fracture intervention trial. J Bone Miner Res 2007;22:503-8.

81

Ishani A, Blackwell T, Jamal SA et al. MORE Investigators. The effect of raloxifene treatment in postmenopausal women with CKD. J Am Soc Nephrol 2008;19:1430-8.

82

Bergner $\mathrm{R}$, Hendrich $\mathrm{D}$, Hoffmann $\mathrm{M}$ et al. Treatment of reduced bone density with ibandronate in dialysis patients. J Nephrol. 2008 Jul-Aug;21(4):510-6.

83

Wetmore JB, Benet LZ, Kleinstuck D et al. Effects of short-term alendronate on bone mineral density in haemodialysis patients. Nephrology (Carlton). 2005 Aug;10(4):393-9. 


\section{Informative Summary}

The incidence of osteoporosis is rising which will result in an increased incidence of fragility fractures in older people. This will lead to a rise in medical visits, admissions to hospitals and a reduction in independent living. Overall this will pose large economic strain on countries and healthcare systems and prevention of such trends is very important. A variety of medical therapies have been developed in recent years to protect against a reduction in bone mineral density and strength in an effort to reduce fracture risk in high risk populations. Fracture risk is assessed using known risk factors, including age and previous fracture, along with dual energy $\mathrm{x}$-ray absorptiometry measurements where available. Clinical risk scores have recently been developed which are validated algorithms used to predict fracture risk over many years. These scores can help to guide treatment decisions and can also be used to assess the cost effectiveness of individual drugs.

Currently the most commonly prescribed anti-resorptive agents include Calcium and Vitamin D and bisphosphonates, which come in a variety of formulations. Bisphosphonates have well documented anti-fracture data and have been in use for many years. However in recent times they have attracted attention due to possible links with oesophageal malignancy, abnormal fragility fractures and osteonecroisis of the jaw. Causal relationships have not been firmly established and continuation of their use as before has been advised by most medicines agencies. Strontium ranelate has a dual mode of action and works by reducing resorption along with increasing bone formation. To date it has provided an alternative first line therapy for patients at high fracture risk or a substitute therapy for those who are unsuitable candidates for bisphosphonates. Parathyroid hormone anabolic therapy given by daily subcutaneous injection offers an effective fast acting treatment for patients with advanced osteoporosis or for patients who fail other therapies. Its use remains quite restricted in many countries due to cost, route of administration and limited data on non-vertebral fracture reduction.

The most recently licensed drug for the treatment of osteoporosis is denosumab, a human monoclonal antibody to receptor activator of NF-kB ligand (RANKL). It is administered subcutaneously twice a year and had shown good vertebral and non-vertebral fracture risk reduction in large international studies. It has been approved for use in the US and Europe 
and is a well tolerated therapy which has shown good levels of patient satisfaction. This exciting new drug is likely to be a very useful alternative therapy in current and future clinical practice. Novel agents such as Odanacatib, a cathepsin K inhibitor and Glucagon-like peptide 2 are currently under investigation for the treatment of osteoporosis and may prove to be interesting future therapies for osteoporosis.

When treating older patients with osteoporosis many factors must be taken into consideration. These include the presence of co-morbidities such as cognitive impairment, gastro-oesophageal disease and chronic kidney disease which can limit the use of some treatments. Compliance difficulties may be an issue with some drug formulations and routes of administration and this should be strongly considered to ensure the most appropriate drug is prescribed to individual older patients with high fracture risk. 


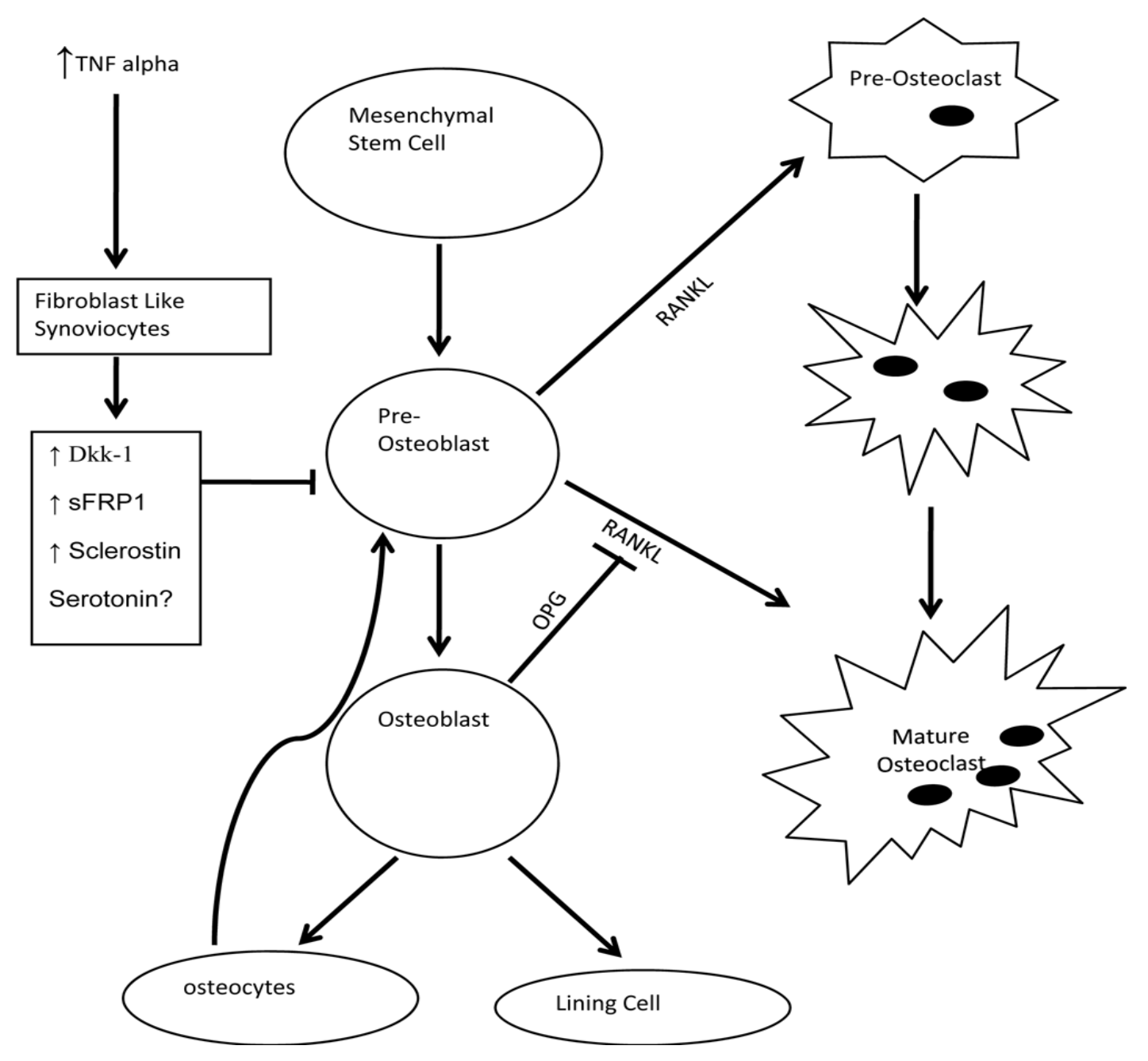




\section{Figure 1.}

Signalling pathways involved in osteogenesis and osteoclastogenesis in bone remodelling. Reproduced with permission of BioMed Central Ltd. Originally published by Lane et al. in Arthritis Research and Therapy 2009,11:228. 


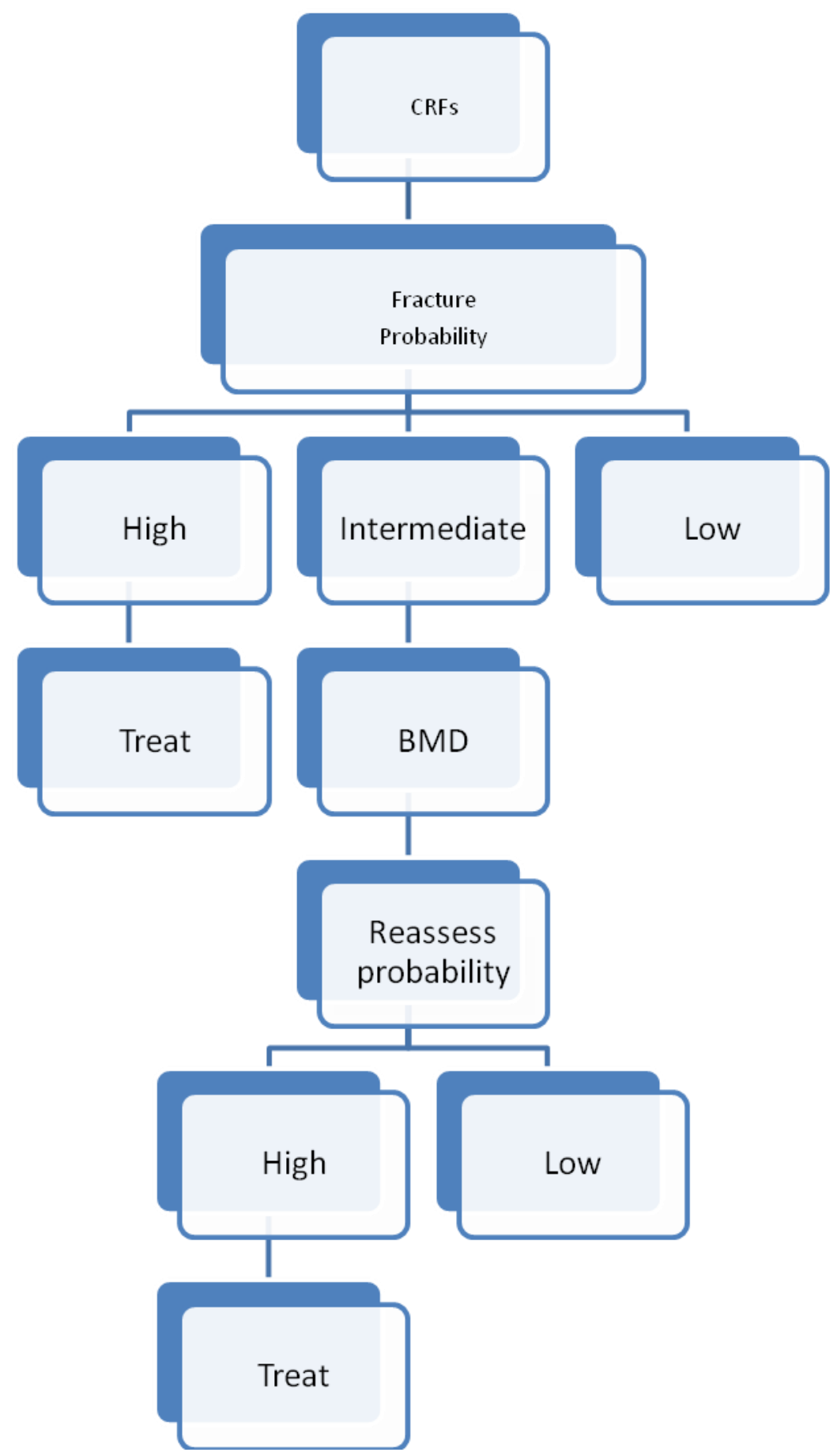

\section{Figure 2.}

Management algorithm for the assessment of individuals at risk of fracture by the use of clinical risk factors and bone mineral density (BMD). Image used with permission of the World Health Organisation Collaborating Centre for Metabolic Bone Diseases, University of Sheffield. FRAX ${ }^{\circledR}$ is registered to Professor JA Kanis, University of Sheffield, UK. Also with kind permission from Springer Science \& Business Media: Osteoporosis International, Development and use of FRAX ${ }^{\circledR}$ in Osteoporosis (2010) 21:(Suppl 2)S407-S413. 
Table 1. Instructions for administration of osteoporosis medications.

\begin{tabular}{|l|l|}
\hline Drug & Administration \\
\hline Bisphosphonates & $\begin{array}{l}\text { Oral bisphosphonates should be taken first } \\
\text { thing in the morning with a full glass of water. } \\
\text { As less than } 1 \% \text { of the dose is absorbed, it is } \\
\text { always taken on an empty stomach. For at } \\
\text { least } 30 \text { minutes after administration eating } \\
\text { and lying down should be avoided. }\end{array}$ \\
\hline Strontium ranelate & $\begin{array}{l}\text { Dissolved in water and given the slow } \\
\text { absorption is taken at bedtime. Calcium } \\
\text { containing foods may reduce bioavailability } \\
\text { by } 60 \text { to } 70 \% \text { and should be avoided for at } \\
\text { least } 2 \text { hours before taking strontium. }\end{array}$ \\
\hline PTH & $\begin{array}{l}\text { Administered as a daily subcutaneous } \\
\text { injection into the thigh or abdominal wall. }\end{array}$ \\
\hline
\end{tabular}

Table 2. Risk factors for osteoporosis.

\begin{tabular}{|l|}
\hline Advanced age \\
\hline Previous fracture \\
\hline Low body weight $(<58 \mathrm{~kg})$ \\
\hline Family history of hip fracture \\
\hline Long term Glucocorticoid therapy \\
\hline Cigarette smoking \\
\hline Excess alcohol
\end{tabular}

